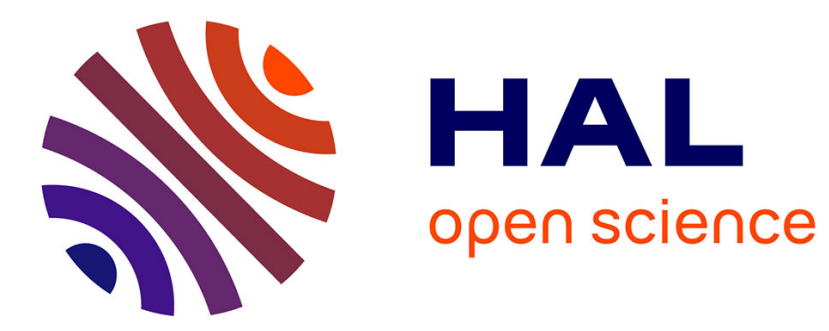

\title{
Le connecteur histoire (de) au regard de ses occurrences dans Frantext
}

Dominique Legallois

\section{To cite this version:}

Dominique Legallois. Le connecteur histoire (de) au regard de ses occurrences dans Frantext. Syntaxe et Sémantique, 2007, 10.3917/ss.008.0061 . hal-03590941

\section{HAL Id: hal-03590941 \\ https://hal.science/hal-03590941}

Submitted on 28 Feb 2022

HAL is a multi-disciplinary open access archive for the deposit and dissemination of scientific research documents, whether they are published or not. The documents may come from teaching and research institutions in France or abroad, or from public or private research centers.
L'archive ouverte pluridisciplinaire HAL, est destinée au dépôt et à la diffusion de documents scientifiques de niveau recherche, publiés ou non, émanant des établissements d'enseignement et de recherche français ou étrangers, des laboratoires publics ou privés. 


\title{
Le connecteur histoire (de) au regard de ses occurrences dans Frantext
}

\author{
Dominique LEGALLOIS \\ Dominique.legallois@unicaen.fr \\ CRISCO (CNRS - FRE 2805) - Université de Caen \\ Article paru dans Syntaxe et Sémantique, 2007, 8, p.61-74
}

\section{Résumé}

La première partie de cet article propose une critique du terme de locution prépositive appliqué au connecteur histoire de + inf. Cette expression ne peut en effet être caractérisée par sa seule fonction prépositive, et la notion de locution ne peut lui être appliquée. La seconde partie propose un examen des occurrences du connecteur dans Frantext (datation, constructions, collocations); à partir d'observations, nous faisons quelques hypothèses sur les origines de la grammaticalisation d'histoire de, et nous posons les caractéristiques sémantiques les plus importantes de la construction.

\section{Abstract}

This paper deals with the connective "histoire de", usually regarded as a complex preposition in both grammatical and lexicological studies. Our contention is that the term "complex preposition" is not relevant: the connective can also work as a conjunction and "de" cannot form a polylexical unit with "histoire". The second part of the paper is a commentary on the observations of the occurrences of histoire de in Frantext (dating, constructions, collocations) ; from these data, we draw up some of the semantic and pragmatic characteristics of histoire de.

Au regard du nombre d'études portant sur les connecteurs, il est assez surprenant de constater que la «locution prépositive ${ }^{1}$ histoire de n'a non seulement jamais fait l'objet d'une étude particulière, mais, plus encore, est très souvent absente des différentes listes de connecteurs dit «argumentatifs », que ces listes soient proposées dans les études sur la notion même de connecteur, ou dans celles sur la locution prépositive, ou encore dans celles sur la grammaticalisation. Ainsi, l'ouvrage récent de Gross et Prandi (2004) dont un des nombreux mérites est de montrer la grande diversité des moyens d'expression de la finalité, ne recense pas ce connecteur, pourtant d'un emploi courant à l'oral.

Cet article ${ }^{2}$ propose donc un examen de histoire de tant au niveau de la caractérisation de son statut catégoriel - qui pose, comme nous le verrons, de multiples problèmes - qu'au niveau de son fonctionnement discursif. Pour ce dernier point, la base Frantext non catégorisée constitue un observatoire bien commode, qui permettra non seulement de dater les premiers emplois de la forme elle-même, mais de repérer également des formes « sœurs » (par exemple l'histoire de rire), d'analyser les contextes énonciatifs, et enfin d'identifier les caractéristiques sémantiques des co-occurrences (essentiellement le prédicat infinitival à

\footnotetext{
${ }^{1}$ Nous verrons que la notion de « locution prépositive » appliquée à histoire de est discutable.

${ }^{2}$ Signalons que cet article fait suite à un précédent (Legallois 2006b), dans lequel il s'agit de déterminer la sémantique du fonctionnement nominal du mot histoire, ainsi que, dans les limites d'un premier aperçu, de poser les prémices d'une analyse du connecteur.
} 
droite de l'expression). La nature de ces co-occurrences se révélera fondamentale pour aborder l'interprétation du connecteur.

\section{Statut catégoriel}

\subsection{Quelle dénomination pour quel statut?}

Histoire de est identifié comme locution prépositive par la plupart des dictionnaires et les rares travaux de linguistique qui mentionnent ce connecteur (par ex. Mélis, 2003). Apparemment, ce statut grammatical ne donne pas lieu à débat, si on suppose qu'une locution prépositive se définit intuitivement comme une suite de mots formant une unité qui remplit en tant que telle la fonction d'une préposition (Mélis, 2003 : 109). On peut dans un premier temps considérer que histoire de est constitué d'une suite polylexicale formant un tout (enregistré comme tel dans les dictionnaires), composée du nominal histoire et de la préposition de. La locution est donnée comme équivalent fonctionnel de pour (par ex. Le Petit Robert - sans qu'il y ait nécessairement réciprocité), même si les exemples ci-dessus montrent une construction différente : une construction «liée » avec pour, une construction détachée avec histoire de:

1- Nous irons au cirque pour nous changer les idées

1 '- Nous irons au cirque, histoire de nous changer les idées

Dans ce qui suit, nous remettons en cause, pour deux raisons, le terme de locution prépositive appliqué comme désignateur de histoire de.

La première raison a déjà été évoquée pour d'autres connecteurs ; par exemple Gross et Prandi (2004) à propos de afin de et de afin que rappellent que la différence entre ces deux formes n'est pas imposée par une nature grammaticale distincte (locution prépositive et locution conjonctive), mais par une contrainte sémantico-grammaticale bien connue : la coréférence ou non entre le sujet de la principale et celui de la subordonnée. De ce fait, il n'y a pas de différence de nature entre afin de et afin que. Le raisonnement vaut évidemment pour histoire de et histoire que $e^{3}$. On perçoit donc une limite à la terminologie classique qui, en s'appuyant sur des critères morphologiques plutôt que fonctionnels, oblitère le fait que nous avons affaire à un même type qui se réalise de deux façons différentes.

Le second point a trait à l'élément «de»dans la composition histoire de. Nous considérons, au vu de la remarque précédente, cet élément comme un complémenteur plutôt que comme une préposition pleine. Mais la question importante est de savoir si ce complémenteur est véritablement constitutif de la locution; ou, dit autrement, si histoire et de forment bien un tout, une locution. Adler (2001) a attiré l'attention sur ce problème à propos de «locutions prépositives » autres que histoire de, en s'appuyant sur une argumentation qui s'applique également ici. Un certain nombre de tests simples appliqués à à cause de, au lieu $d e$, en dépit de montrent que de ne peut véritablement être considéré comme faisant partie de ces locutions : il est seulement sélectionné, «de même que les verbes transitifs indirects ou les adjectifs dits "transitifs» (apte à, capable de) sélectionnent leur préposition, et à travers elle, régissent un complément »(Adler, 2001 : 162). Ainsi, la coordination (ex. 2 et 2'), l'emploi d'une expression extraprédicative (ex. 3 et 3'), ou encore l'alternance avec que selon la coréférence ou non entre les deux sujets.

\section{2- Il a réussi en dépit de sa maladie et de son état moral (ex. d'Adler)}

\footnotetext{
${ }^{3}$ Il n'y a que cinq occurrences de histoire que $P$ dans Frantext.
} 
2'- Cette dernière voiture s'arrêta sur la route, Tron ayant accompagné l'autre jusqu'au parc, à travers le chaume, sous le prétexte de donner un coup de main : histoire de flâner et de causer un instant. E. ZOLA, La Terre, 1887

\section{3- Il a réussi en dépit, d'ailleurs / dit-il, de sa maladie (ex. d'Adler)}

3'- Cette dernière voiture s'arrêta sur la route, Tron ayant accompagné l'autre jusqu'au parc, à travers le chaume, sous le prétexte de donner un coup de main : histoire, d'ailleurs / dit-il, de flâner et de causer un instant

les exemples ci-dessus indiquent que «de » n'est pas soudé, mais seulement gouverné. De là, on ne peut le considérer raisonnablement comme élément de la locution. Si la notion de locution peut rester pertinente pour en dépit, au lieu, etc., en raison de la première préposition qui, elle, reste soudée (mais cette perspective n'est pas celle de Adler qui a une conception de la locution comme construction figée à des degrés divers - l'auteur préfère donc parler de prépositions simples pour à cause, au lieu, en dépit), elle n'est plus du tout pertinente pour histoire : difficile, en effet, de considérer le morphème histoire comme une locution.

On voit bien le problème terminologique que nous rencontrons : d'une part, le terme de préposition, ou même l'adjectif prépositive sont trop restrictifs dans la mesure où, au regard de l'emploi conjonctif, on doit parler de réalisations différentes d'un même type ; d'autre part, la notion de locution n'est plus pertinente. Nous parlerons donc simplement de connecteur, avec, évidemment, les inconvénients bien connus d'un emploi trop général.

\subsection{Finalité ou causalité ?}

Sans surprise, le discours lexicographique voit en histoire $(d e)^{4}$ un introducteur de proposition finale. Mais l'idée de finalité inhérente à histoire (de) semble insuffisante. Ainsi, le Dictionnaire du Français usuel de Picoche et Rolland, introduit la notion de justification :

Histoire de + verbe à l'infinitif (fam.) : explication donnée par A1 à A2 pour justifier une action. J'ai agi ainsi, histoire de voir comment tu réagirais.

Explication et justification sont des relations discursives relevant généralement de la cause ; par exemple, J.Hobbs (1990) fait de l'explication un cas de relation causale. Mais on peut très bien concevoir qu'une visée (le procès $\mathrm{Y}$ dans $X$, histoire de $Y$ ) serve d'explication à un acte $X$. Nous adopterons la catégorie aristotélicienne ${ }^{5}$ de cause finale pour caractériser la valeur de cohérence entre Y et X. En effet, comme nous l'avions montré dans Legallois (2006b), la valeur intentionnelle de $\mathrm{X}$ et la constitution de $\mathrm{Y}$ comme procès à réaliser (cf. les formes infinitivales ou le subjonctif ${ }^{6}$ ) doivent être compris selon le schéma téléologique suivant

$L$ 'intention de faire $Y$ est à l'origine de $X$.

D'où l'idée que $Y$ est bien une motivation en même temps que la cible de X. Ce schéma s'applique évidemment à d'autres connecteurs finaux, mais permet de préciser le type de finalité dont il est question ici.

\subsection{Caractéristiques}

\footnotetext{
${ }^{4}$ Nous adoptons désormais cette notation. pour indiquer que de n'est pas intégré.

${ }^{5}$ Cause finale, cause matérielle, cause efficiente et cause formelle forment la notion de $\alpha \iota \tau ı o v$ [aition] dans la Métaphysique d'Aristote.

${ }^{6}$. Les locutions conjonctives qui servent à construire les propositions finales entraînent l'emploi du mode subjonctif dans la mesure où elles explicitent cette intention (Wagner et Pinchon, 1962, § 699).
} 
Si on considère bien histoire (de) comme un mot grammatical, c'est en raison uniquement de son équivalence fonctionnelle avec les prépositions pour (recouvrement partiel), afin de, dans l'objectif de, dans le but de, dans l'intention de (recouvrement total), et non pour son refus d'une modification adjectivale ou de la flexion du pluriel, puisque certains emplois nominaux observent la même contrainte ${ }^{7}$; par exemple :

\section{4- Dans la (* longue) histoire, j'ai oublié mes clefs chez Jacques}

5- C'est une (* petite) histoire de minutes

Mais histoire (de) est manifestement un hapax grammatical. Il est en effet peu envisageable de rapprocher sa forme d'une autre expression. Les prépositions nominales, par exemple, ont un fonctionnement discursif différent (thématisation (6), caractérisation (7)) et sélectionnent un nom

6- Question / point de vue / côté science fiction, il en connaît un rayon

7- Un film genre / style James Bond

Faute de + inf. possède une forme proche : détachement et construction infinitive. Néanmoins, la construction faute que + subj. ne semble pas recevable, et histoire (de) ne peut être suivi par un nom (sauf l'exemple atypique de 9)

On pourrait également comparer histoire (de) au connecteur crainte (de / que) : il est composé d'un élément nominal sans déterminant, il gouverne une proposition, il est construit par détachement. Mais quelques traits plus ou moins décisifs l'en distingue: comme le remarquent Gross et Prandi (2004), crainte (de) est d'un emploi littéraire, alors que histoire (de) est recensé comme familier ; il y a effacement (il s'agit en fait du cas le moins fréquent) d'une préposition avant crainte (de / que) : par crainte (de) / de crainte (de); histoire (de) ne peut être précédé d'une préposition. De plus crainte (de) connaît la concurrence de la locution apparentée dans la crainte de :

8- Les français se sont désistés, dans la crainte de dépenses excédant leurs faibles revenus (ex. de Gross et Prandi, 2004),

Histoire (de) ne peut connaître une telle concurrence. Crainte (de) peut introduire un GN, ce qui n'est pas possible pour histoire (de), excepté cet emploi particulier et unique (à notre connaissance) relevé par le TLF :

9- J'ai remis chez vous, en allant au chemin de fer, vos deux volumes non enveloppés, histoire de la grande précipitation où j'étais (P.MERIMEE, Lettre à une Inconnue, t. 2, 1858).

On peut considérer cet emploi purement causal comme un calque de faute de $+N /$ crainte de $+N$.

Autre différence, morphologique celle-ci, crainte est un déverbal, ce qui n'est pas le cas d'histoire ; de plus, crainte reste sémantiquement transparent (Gross et Prandi (2004) classent crainte (de) parmi les prédicats finaux de sentiment), alors que la signification de histoire est opaque dans cet emploi.

Nous concluons de ces observations rapides que histoire (de) constitue un hapax grammatical ${ }^{8}$.

\footnotetext{
${ }^{7}$ On devrait évoquer la non détermination de histoire, contrairement à l'objectif, le but, l'intention et même à la seule fin de.

${ }^{8}$ Hapax...pas tout à fait ! A l'heure où nous bouclons, notre collègue Mathilde Salles nous signale la présence de la forme question de + inf. dans les romans de Céline ; par exemple : Je l'écoutais battre son coeur, question de faire quelque chose dans la circonstance, les quelques gestes qu'on attendait (Céline, Voyage au bout de la
} 


\section{Observation des occurrences dans la base Frantext}

Nous avons procédé au recensement exhaustif des 372 emplois du connecteur dans la base non catégorisée de Frantext. Ce recensement permet non seulement la datation des premiers emplois, mais également de proposer quelques hypothèses sur la grammaticalisation du connecteur.

\subsection{Datation des premiers emplois}

La forme du premier emploi identifié est assez surprenante : l'histoire de rire, 1831, avec donc une détermination ;

10- C'était, mon lieutenant, l'histoire de rire... pour lors j'en arrête une par les cheveux et je l'embrasse... E. SUE, Atar-Gull, 1831

On trouve deux autres fois dans Frantext ${ }^{9}$ cette même forme à la même époque, dans le même contexte (des avances plus ou moins prononcées faites à des femmes) et dans la même construction ; toujours chez Sue en 1843 :

11-... si Alfred savait cela?

Ne m'en parlez pas, le sang me tourne rien que d'y songer. Alfred est jaloux comme un Bédouin; et pourtant, de la part du père Joseph, c'est l'histoire de rire, en tout bien, tout honneur. E. SUE, Les Mystères de Paris, 1843

Mais également chez Sand (1844) :

12 - Ce baiser sur la main ne t'a pas offensée?

- Oh! Je voyais bien que ce monsieur ne voulait pas m'offenser ; c'était l'histoire de rire. G. SAND, Jeanne, 1844

On ne peut faire ici que des conjectures: soit, il s'agit d'une modification idiosyncrasique du connecteur histoire (de), et une remotivation du statut nominal de histoire. Cela démontrerait que histoire (de) est déjà présent dans le discours oral populaire avant 1830 , sans être toutefois tout à fait stabilisé. Soit ces extraits témoignent d'une étape dans la grammaticalisation, la forme l'histoire de + infinitif désormais disparue, dont nous n'avons que quelques attestations. Seul un examen plus précis de la littérature populaire des années 1820-1830 ${ }^{10}$ pourrait être éclairant, et permettrait d'argumenter en faveur de telle ou telle hypothèse.

Remarquons que la notion de grammaticalisation est, pour le cas de histoire (de), sans doute mal appropriée puisqu'il est impossible d'observer avec certitude une forme libre constituant la base de cette grammaticalisation.

Deux autre occurrences, plus tardives, ont été repérées; toujours dans le même contexte :

nuit ). Après examen sur Frantext, nous n'avons trouvé ce type d'emploi - concurrent de histoire de - que chez cet auteur.

${ }^{9}$ En plus de l'ex.16, plus bas, mais qui apparaît dans une forme non clivée.

${ }^{10}$ Mais on imagine bien que sans numérisation, la tâche est colossale. 
13- ce n'est pas tant l'histoire de regarder les femmes. Chez nous autres, on peut être sauvé malgré les femmes. Un rabbin peut avoir une femme. G. DUHAMEL, Le jardin des bêtes sauvages, 1934

Ou dans un autre :

14 -si le vent ne fraîchit pas trop, je viendrai peut-être vous réveiller cette nuit, pour l'histoire de rire, dit-elle. G.BERNANOS, Un crime, 1935

Ces emplois, certes très circonstanciés, pourraient avoir le mérite d'exhumer le passage d'une forme nominale figée à la forme grammaticale, ainsi, d'ailleurs, que d'exhiber la tension entre les deux formes. L'exemple de Bernanos, difficile à analyser en raison de son idiomaticité, semble ressusciter la préposition causale pour, et plaiderait ainsi en faveur d'un effacement double (préposition et article) dans le processus de grammaticalisation qui conserverait la trace de la causalité. Cependant, les exemples beaucoup plus anciens de Sue et Sand, donc plus près de l'origine du connecteur, ne sont pas construits avec pour. En fait, dans Legallois (2006b), nous avons montré que l'idée de causalité, mais aussi de finalité, est inhérente au morphème histoire. Un double marquage apparaît donc redondant.

Par ailleurs, dans le roman de F. Soulié Les mémoires du diable (1837), on peut éventuellement miser sur un indice en faveur de l'apparition de la construction histoire (de) vers les années 1820-1830: non seulement parce que le premier emploi sans détermination est identifié dans ce roman de 1837 ainsi que dans César Birotteau (1837) de Balzac, mais aussi parce qu'à plusieurs reprises, un personnage qui a vendu son âme au diable, prononce avant ses méfaits, l'expression histoire de rire. Elle constitue, comme le précise à quatre reprises le narrateur, un infatigable refrain, un fameux mot qui devient diabolique :

15- ce mot rebutant qu'il jette comme moralité au bout de toutes ses actions; ce mot : histoire de rire! est souvent aussi sombre que le mot du trappiste : frère, il faut mourir! (F. SOULIE, Les mémoires du diable, 1837)

On peut penser que l'expressivité de ce «mot» prononcé après que le personnage a produit des mauvaises actions, est redevable à son emploi sarcastique et sardonique, mais également à sa récence, à un moment où la grammaticalisation a certes opéré mais où la nouveauté surprend encore. Là encore, évidemment, il s'agit d'une hypothèse de lecture. Nous notons encore l'usage (unique) dans ce roman, de la forme

16- Cependant toutes les farces de cet homme n'ont pas eu pour but une vengeance; l'histoire de rire est le grand principe de ses tours. F. SOULIE, Les mémoires du diable, 1837

qui témoigne spectaculairement de la motivation nominale de la forme grammaticale. Le terme principe ici s'accorde bien avec l'idée relevée plus haut d'une cause première, donc d'une cause finale.

\subsection{Contextes énonciatifs}

Tous les premiers emplois apparaissent dans du discours direct, et ont pour locuteurs des personnages "du peuple » s'exprimant dans un français familier. Notons également que ces occurrences se situent dans le roman populaire feuilletonesque (Sue, Soulié), ou bien dans le roman réaliste (Balzac), et non dans la littérature romantique de la même époque (aucun emploi de histoire (de) chez Stendhal ou chez Hugo ${ }^{11}$ ). Tout cela atteste, s'il en est, du caractère familier et oral de l'expression. Sauf erreur de notre part, le premier emploi identifié

\footnotetext{
${ }^{11}$ On trouve une occurrence chez Hugo dans Actes et Paroles III
} 
dans la narration avec un narrateur extradiégétique (dans une narration sans «je ») - et non plus dans le discours direct- date de 1877, dans l'Assommoir de Zola (à sept reprises). Par exemple :

17- Le soir même, le zingueur amena des camarades, un maçon, un menuisier, un peintre, de bons zigs qui feraient cette bricole-là après leur journée, histoire de rendre service. E. ZOLA, L'Assommoir, 1877

On constate que ces occurrences sont employées dans le discours indirect libre (Dil). Le Dil, en plein essor à cette époque, constitue, en ce qui concerne le texte écrit, un procédé puissant pour une promotion du connecteur: il permet la transition d'un emploi oral, représenté dans les dialogues, vers des emplois de plus en plus indépendants du dialogal : d'abord dans le Dil, où la voix énonciative est encore celle d'un personnage, puis dans la narration ou l'énonciateur est le narrateur extradiégétique. On peut penser que ce nouvel usage témoigne d'une diffusion massive et d'une bonne intégration du connecteur dans la deuxième moitié du $19^{\mathrm{e}}$ dans les écrits romanesques.

\subsection{Détachement}

Mis à part quelques cas de ponctuation particulière et la structure clivée qui procède par elle-même à une sorte de détachement (c'est histoire de rire), toutes les occurrences recensées se manifestent dans des constructions détachées ${ }^{12}$. Le détachement est le plus souvent matérialisé par une virgule, mais aussi par les parenthèses, les deux points, une phrase isolée.

La construction détachée est l'indice d'une prise en charge énonciative ; on peut la considérer comme une représentation iconique d'un décrochage énonciatif. En effet, le détachement permet de mimer une pause délibérative, un moment de réflexion conduisant à re-évaluer a posteriori $\mathrm{X}$ comme un procès intentionnellement orienté vers la réalisation de $\mathrm{Y}$, d'où l'effet de justification ou d'explication selon les contextes. Il ressort de cette observation que la responsabilité de la justification / explication par la cause-finale incombe au locuteur, et non au référent sujet de la phrase (sauf bien sûr, si les deux coïncident).

\subsection{Les co-occurrents}

Outre le détachement, un des points fondamentaux à souligner est le type de prédicat à l'infinitif. Ainsi, on observe que sur les 372 emplois, le verbe intransitif rire (et ses synonymes) apparait 64 fois $^{13}$, voir intransitif (et ses synonymes) 18 fois, causer intransitif (et ses synonymes) 17, passer le temps (et ses synonymes) 18 et boire / manger 13. Même si moins fréquentes, d'autres co-occurrences sont observables (se dégourdir les jambes, souffler...).

\subsubsection{Histoire de rire et les autres prédicats « inconsistants » : Y comme intention limitée.}

Histoire de rire ${ }^{14}$ est employé de façon massive ; on peut considérer qu'il s'agit d'une collocation (à l'entrée rire le Petit Robert et le Trésor de la Langue française informatisé donnent histoire de rire). Incontestablement, tous les premiers emplois du connecteur sélectionnent le verbe rire et constituent une alternative à pour rire, une autre collocation

\footnotetext{
1251 occurrences présentent un détachement frontal, place privilégiée pour une portée plus grande du circonstant et une fonction d'organisation textuelle. Malgré tout, après examen, il est difficile de dire que histoire (de) a une portée au-delà de la phrase.

${ }^{13}$ Le roman de Soulié mentionné ci-dessous à recours, à lui seul, 17 fois au verbe rire. Même en pondérant, rire est de loin le prédicat le plus employé.

${ }^{14}$ On ne doit pas sous estimer la motivation «anthropologique » de la grammaticalisation de histoire. Les histoires sont souvent racontées pour rire, pour passer le temps.
} 
(pour de rire apparait dans Frantext chez E.Sue, en 1845, soit approximativement à la même époque que histoire (de)). Dans cet emploi, rire (mais aussi les autres co-occurrents récurrents - boire/manger, voir, passer le temps, etc.) constitue ce que nous appellerons un procès « inconsistant », c'est à dire un procès qui ne possède pas d'effets ou de conséquences notoires. D'où cette valeur : si le procès est inconsistant, l'intention qui est à son origine ne peut être elle-même qu'une intention simple. Ainsi, justifier l'action X par l'intention de réaliser un procès inconsistant, permet de circonscrire $\mathrm{X}$ dans un cadre limité, sans effets particuliers.

Nous pourrions aller jusqu'à dire que dans certains emplois, l'énonciateur n'essaye pas véritablement de donner les explications ou justifications d'un procès, mais qu'il les donne tout de même, en manifestant cependant par l'emploi de motifs stéréotypés son indifférence ou son détachement envers cette justification. C'est ce que montre, selon nous, l'emploi avec un complément $\varnothing$ de 25 (cf. plus bas) fréquent à l'oral.

Notons encore qu'un complément « inconsistant» est également une valeur présente dans certains emplois nominaux figés :

18- C'est une histoire de minutes

19-C'est l'histoire d'une minute ou deux

20-C'est l'histoire d'un ou deux couverts de plus

la quantification est nécessairement vue comme négligeable, peu «consistante », dans les emplois qu'il faudrait rapprocher de 10, 11, 12 .

Par ailleurs, l'emploi relativement fréquent (40/367) d'un quantificateur « faible » ou d'un déterminant indéfini est tout à fait congruent avec la valeur d'inconsistance. Par exemple :

21- Histoire de prendre un peu l'air, je suis allé manger un morceau en ville. Ph. DJIAN Philippe, $37^{\circ} 2$ le matin, 1985

22- Il paraît qu'il s'était mis en colère une seule fois, mais de cette colère contre un voisin qui avait déplacé les bornes d'un champ, histoire de gagner quelques mètres. J.LANZMANN, Le Têtard, 1976

23- Cette dernière voiture s'arrêta sur la route, Tron ayant accompagné l'autre jusqu'au parc, à travers le chaume, sous le prétexte de donner un coup de main : histoire de flâner et de causer un instant. E. ZOLA, La terre, 1887

24- Histoire de lui acheter quelque chose, Pierre acquiert pour sa soeur quelques images pieuses, imprimées au temps des combats. J. ROUAUD, Les Champs d'honneur, 1990

25- Il attendit pour voir si un con en treillis rirait de son esprit. Il lui aurait fait faire une petite marche de nuit, histoire de. J. VAUTRIN, Bloody Mary, $1979^{15}$

\subsubsection{Effets de sens}

Bien sûr, tous les arguments ne réfèrent pas nécessairement à des procès inconsistants. Dans Frantext, la complémentation par des procès à la fois «non intrinsèquement inconsistants »et non récurrents, apparaît dès 1840 :

26- J'ai soutiré douze francs à votre beau-père, les voilà...

- Et comment as-tu fait?...

- Ne voulait-il pas voir les bassines et les provisions de monsieur, histoire de découvrir le secret. Je savais bien qu'il n'y avait plus rien dans la petite cuisine; mais je lui ai fait peur comme s'il allait voler son fils, et il m'a donné deux écus. H. BALZAC, Illusions perdues, 1843.

Découvrir le secret n'est pas par lui-même inconsistant, mais, en tant que complément de histoire (de), il est malgré tout construit par l'énonciation comme objet d'une lubie, d'un caprice, c'est-à-dire d'une intention bien réelle de la part du beau-père, mais déconsidérée par le locuteur. Les écrivains ont su jouer de cette pression de la forme sur le complément phrastique ; mais d'abord, considérons deux exemples (construits) qui paraissent incongrus ou particulièrement cyniques :

${ }^{15}$ Exemple qui contredit Mélis (2003: 114) qui affirme que histoire (de) et à cause de nécessitent obligatoirement un complément (cela est seulement exact pour à cause de). On parlera, pour cet exemple, d'un complément $\varnothing$. 
27- Hitler a armé l'Allemagne, histoire d'envahir l'Europe

28- Paul a pris des médicaments, histoire de se suicider

Envahir l'Europe ou se suicider sont des actions ayant des conséquences particulièrement graves, qui ne peuvent, par conséquent, être considérées comme les objets d'une simple intention. Parmi les nombreux exemples de ce type dans Frantext, on relèvera :

29- Il en prit une, l'alluma et tira dessus comme un crapaud, histoire de salir un peu plus ses bronches (A. Jardin, Bille en tête, 1986)

Se salir un peu plus les bronches est certes un procès consistant par lui-même, puisque occasionnant des effets dévastateurs; histoire (de) configure ce procès comme ne possédant pas de conséquences notoires - d'où l'effet humoristique quelque peu corrosif. Mais il y a plus, est construit ici un rapport intentionnel entre tirer dessus comme un crapaud et se salir les bronches, rapport qui n'existe pas objectivement, mais qui est le fait de l'énonciateur. De même, dans

30- J'ai jeté un coup d'œil un peu triste sur les baraques et je me suis coltiné un bidon de vingt-cinq kilos le long du chemin, histoire de me cisailler un peu les doigts (Ph. Djian, 37² le matin, 1985).

une relation intentionnelle incongrue est imposée par la forme.

Enfin :

31- Mais, avec une malice appuyée, il se disait néanmoins séduit par les mouvements en cours : histoire de montrer qu'il restait jeune et de gauche (J. Kristeva, Les Samourais, 1990)

« Montrer que l'on reste jeune et de gauche » n'est pas intrinsèquement sans valeur, mais est ici configuré comme tel. Cette inconsistance est en fait un jugement de l'énonciateur (ici le narrateur), jugement négatif d'ailleurs anticipé par avec une malice appuyée, et qui façonne l'ethos du personnage : quelqu'un de puéril, sur le retour et ayant perdu ses idéaux. C'est le regard du narrateur qui est ironique ici, et non pas le rapport entre l'intention de faire $\mathrm{Y}$ et celle de faire $\mathrm{X}$ (comme dans 30 ).

\subsection{Le procès $X$ dans la limite d'une configuration}

L'examen des procès inconsistants permet donc de comprendre les effets pragmatiques de histoire (de). Mais les remarques faites jusqu'à maintenant n'expliquent sans doute pas les motivations de l'emploi du mot histoire.

Même si la notion d'expressivité est incontestablement peu objective, elle permet de rendre compte de l'impression ressentie à la lecture des exemples : histoire (de) est plus « coloré » que pour; ainsi 29 comparé à

32- Il en prit une, l'alluma et tira dessus comme un crapaud, pour se salir un peu plus ses bronches ;

est bien plus expressif dans la mesure où histoire de, par rapport à pour en dit « un peu plus ». Cette expressivité pourrait s'expliquer ainsi : en étant attentif au fonctionnement nominal (Legallois, 2006b), on peut observer que le nom est utilisé pour circonscrire un ensemble cohérent d'événements qui ont pour seule épaisseur ontologique leur participation à une finalité précise. Ils sont orientés vers une seule fin ; suivant en cela la narratologie, il est possible de désigner cette fonction par le terme de configuration. Cette configuration peut être un ensemble d'événements vus comme cohésifs, toujours orienté vers la réalisation d'un objet (un devenir) ; il s'ensuit que tout événement de la configuration n'a pas d'autre réalité (c'est- 
à-dire pas d'autres effets) ailleurs que dans ce cadre. Des emplois nominaux jouent argumentativement sur cet aspect « fermé sur elle-même » de l'histoire

33 - Ce ne sont pas tes histoires! (N'interviens pas là-dedans)

34 - Tes histoires n'intéressent personne

35 - N'aie pas peur, ce n'est qu'une histoire

36 - Je ne crois pas à ce qui vient d'arriver à Paul! C'est une histoire de fou!

37 - Je n'entre pas dans cette histoire là (dans le sens, je n'entre pas dans ces considérations / dans la combine)

A chaque fois, ces expressions relativement figées, exploitent le potentiel sémantique de histoire: configurer des événements et leur refuser toute réalité en dehors de cette configuration. De même, histoire (de) met en jeu cette valeur : dans $X$, histoire de $Y$, le procès $\mathrm{X}$ est dit justifié par sa seule finalité, "réaliser $\mathrm{Y}$ » (d'où la valeur restrictive soulignée plus haut). Ainsi, 32, tout en possédant les mêmes effets, est moins expressif que 29 car pour ne dit pas ce que dit histoire: Il en prit une, l'alluma et tira dessus comme un crapaud, est conceptualisé comme ensemble de procès limités à un cadre / configuration, à un scénario en dehors duquel cet ensemble n'aurait aucune raison d'être.

\section{Conclusion}

Nous récapitulons ici nos observations : histoire (de), qui peut difficilement être considéré comme une locution en raison du comportement de de, est un connecteur apparaissant dans la littérature vers 1837 - peut-être comme grammaticalisation et généralisation d'une première forme : l'histoire de rire, mais les données sont trop insuffisantes pour que nous soyons affirmatifs. Son caractère expressif est l'héritage d'un emploi oral populaire. Employé systématiquement en construction détachée, il introduit un procès conçu comme une cause finale servant à justifier ou à expliquer le recours à une action. Le point de vue exprimé par histoire (de) est subjectif, produit par l'énonciateur et non par le référent du sujet phrastique.

La conjonction sélectionne d'abord (chronologiquement et statistiquement) le prédicat rire, à une époque où apparaît également pour de rire et où pour rire constitue déjà une expression idiomatique. Le trait inconsistant de ce verbe (et d'autres co-occurrents) révèle que le procès $\mathrm{X}$ est motivé par une intention "simple » (la réalisation de Y), et qu'il n'a pas d'autres effets en dehors de cette configuration. Ainsi, nous pouvons considérer histoire de + inf / que $P$ comme une construction, au sens des Grammaires de Constructions ${ }^{16}$, c'est-à-dire une forme phraséologique mi-lexicale, mi-grammaticale, à laquelle est inhérente une valeur sémanticopragmatique.

\section{Références}

ADLER S., 2001, «Les locutions prépositives: questions de méthodologie et de définition », Travaux de Linguistique, $\mathrm{n}^{\circ} 42-43$ : 157-170

FILLMORE C.J, KAY P. \& O'CONNER M.C, 1988, «Regularity and idiomaticity in grammatical constructions: the case of let alone», Language 64, 501-38.

GOLDBERG A.E. (1995) Constructions: A Construction Grammar Approach to Argument Structure, University of Chicago Press

GROSS G. et PRANDI M., 2004, La finalité: fondements conceptuels et genèse linguistique, Bruxelles, De Boeck, Duculot

GROSS G., 1996, Les expressions figées en français : noms composés et autres locutions, Gap, Ophrys

\footnotetext{
${ }^{16}$ Cf. A. Goldberg (1995), Ch. Fillmore et ali. (1988) pour la construction let alone (cf. E. Roussel ici même), D.Legallois et J. François, (2006a).
} 
HOBBS J. R., 1990. Literature and Cognition, Lecture Notes, Number 21, Center for the Study of Language and Information, Stanford, California.

LEGALLOIS D., et FRANÇOIS J., 2006a, «Autour des Grammaires de Construction et de Patterns », Cahier du CRISCO n $^{\circ}$ 21, téléchargeable sur http://www.crisco.unicaen.fr

LEGALLOIS D., 2006b, « Détermination sémantique, contraintes syntagmatiques, histoire de comprendre (un peu) le figement linguistique » in La composition syntaxique et le figement lexical, Bibliothèque de Syntaxe et Sémantique 2, Presses Universitaires de Caen.

MELIS L., 2003. La préposition en français, Gap, Paris, Ophrys

WAGNER R.L. et PINCHON J., 1962, Grammaire du Français classique et moderne, Paris, Hachette Supérieur 